\title{
Optimal Design for MIMO Relay System
}

\section{Fangni Chen}

Department of Communication and Electronic Engineering, Zhejiang University of Science and Technology, Hangzhou, China. Email: cfnini@163.com

Received May, 2013.

\begin{abstract}
Recently, multiple-input multiple-output (MIMO) relay technique has been received great attention due to its prominent ability to provide broad coverage and enhance the link reliability and spectral efficiency. In this paper, an overview of optimal design for single user and multiuser non-regenerative MIMO relay systems is proposed. We explore some key designs of source node and destination node as well as relay node processing matrices using minimum mean square error (MMSE) criterion under the transmit power constraints. Simulation results compare different methods in terms of the MSE and bit error rate (BER) performance.
\end{abstract}

Keywords: MIMO; Relay; Optimal Design; MMSE

\section{Introduction}

Multiple-input multiple-output (MIMO) relay communication, which incorporates relaying technology in MIMO network, has attracted considerable attention in recent years due to its potential ability to extend network coverage and improve link reliability as well as spectral efficiency. The aim of this paper is to provide an overview of optimal design for single user non-regenerative MIMO relay systems.

A MIMO relay can be regenerative or non-regenerative, full-duplex or half-duplex, one-way or two-way [1]. A regenerative relay requires digital decoding and reencoding at the relay, which can cause a significant increase of delay and complexity. A non-regenerative relay does not need any digital decoding and re-encoding at the relay, which is a useful advantage over regenerative relays. The difference between full-duplex relay and halfduplex relay depends on whether relays can transmit and receive in the same time or same frequency or not. Fullduplex relay is spectrally efficient but causes a problem of self-interference and half-duplex relay is easy to implement but not spectrally efficient as full-duplex. The difference between two-way relay and one-way relay is whether relays can relay information in two directions in a single time or single frequency or not. In this paper we will focus on non-regenerative, half-duplex, one-way MIMO relay systems.

Initial research on MIMO relay began in single user [2], where the capacity bounds of MIMO relay systems were examined. It has been shown in [3] and [4] that performing linear processing at the relay node can out- perform the conventional AF relaying in a non-regenerative, also known as amplify-and-forward (AF), MIMO relay system. The optimal relay amplifying matrix which maximizes the mutual information (MI) between source and destination is derived in [5]. In [6], a minimum mean square error (MMSE)-based iterative algorithm is proposed for jointly designing the source, relay and destination processing matrices. A unified framework is developed in [7] to jointly optimize the source preceding matrix and the relay amplifying matrix for a broad class of objective functions.

In this paper, we focus on optimization for single user non-regenerative MIMO relay systems where each node is equipped with multiple antennas supporting multiple data streams. We explore two design schemes to optimize the transmitter and receiver aiming at minimizing the MSE. First we introduce an iterative algorithm to find the optimal source preceding matrix and relay amplifying matrix. Considering the complexity in practical applications, we also introduce the simplified algorithm to solve the same optimization problem. Simulation results show the simplified algorithm has a comparable performance with the iterative one.

The rest of this paper is organized as follows. In Section II, we introduce the single user non-regenerative MIMO relay system model and formulate the optimization problem. Different optimization methods of obtaining the source preceding matrix and relay amplifying matrix structure under power constraints are analyzed in section III. Simulation results are conducted to compare the system performances of the different methods in Section IV. Finally, extensions and future work are drawn in 
Section V.

Notations: boldface letters represent matrices and vectors. $(\cdot)^{H}$, blkdiag $(\cdot)$, I and $\operatorname{tr}(\cdot)$ stand for conjugate transpose, the block diagonal matrix composed of $(\cdot)$, the identity matrix and the trace, respectively. $e[\cdot]$ indicates the statistical expectation.

\section{System Model}

Consider a single user non-regenerative MIMO relay system model, which is shown in Figure 1. This model supports one source node (BS) transmitting the symbols to one destination node (MS) and one relay node (RS) assists the course of transmission between them, where the BS, RS and MS have $N_{s}, N_{r}$ and $N_{d}$ antennas, respectively. The direct links between the BS and the MS is neglected in order to simplify the analysis. The transmission is comprised of four time slots. In the first time slot, the $M \times 1$ data stream vector $\mathbf{x}$ is linearly precoded at the BS by a $N_{s} \times M$ source preceding matrix $\mathbf{T}$. Then the preceded vector is transmitted to the RS, the received signal at the RS can be written by

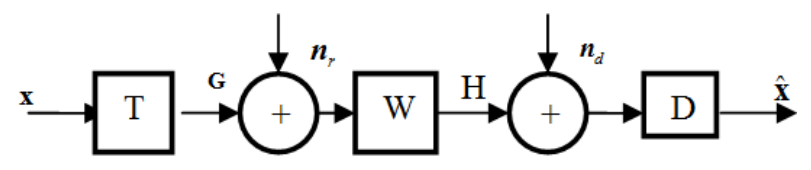

Figure 1. Single user MIMO relay system model.

$$
\mathbf{y}_{r}=\mathbf{G T x}+\mathbf{n}_{r}
$$

where $\mathbf{G}$ is the $N_{r} \times N_{s}$ MIMO channel matrix between the source and the relay, $\mathbf{n}_{r}$ is the $N_{r} \times 1$ additive Gaussian noise vectors with $e\left[\mathbf{n}_{r} \mathbf{n}_{r}^{H}\right]=\mathbf{I}$ at the relay node. For a linear non-regenerative MIMO relay system, there should be $M \leq \min \left(N_{s}, N_{r}, N_{d}\right)$, otherwise the system can not support active $M$ symbols in each transmission.

In the second time slot, the BS remains silent and the RS multiplies (linearly amplifies) the received signal vector $\mathbf{y}_{r}$ by a $N_{r} \times N_{r}$ relay amplifying matrix $\mathbf{W}$ and transmits the amplified signal vector to the MS. Hence the received signal vector at the MS can be written as

$$
\mathbf{r}=\mathbf{H W G T x}+\mathbf{H W} \mathbf{n}_{\mathbf{r}}+\mathbf{n}_{d}
$$

where $\mathbf{H}, \mathbf{r}$, and $\mathbf{n}_{d}$ are the $N_{d} \times N_{r}$ MIMO channel matrix between the RS and the MS, the received signal and the additive Gaussian noise vectors at the MS, respectively. Linear receiver is used at the MS to recover the signals. Thus the estimated vector is eventually given by

$$
\begin{aligned}
\hat{\mathbf{x}} & =\mathbf{D}\left(\text { HWGTx }+ \text { HWn }_{r}+\mathbf{n}_{d}\right) \\
& =\mathbf{D}(\text { HWGTx }+\mathbf{n})
\end{aligned}
$$

where $\mathbf{D}$ is the $M \times N_{d}$ weight matrix. $\mathbf{n}=\mathbf{H W n _ { r }}+\mathbf{n}_{d}$ is equivalent noise vector. We assume that the channel matrices $\mathbf{G}$ and $\mathbf{H}$ are all quasi-static and known to the BS, RS and MS through channel estimate method. We also assume that all noises are independent and identically distributed (i.i.d.) complex circularly symmetric Gaussian noise with zero mean and unit variance. Another two time slots are needed for the reverse link.

Follow the system model above, we can get the mean square error matrix

$$
\begin{aligned}
\mathbf{E} & =e\left[(\mathbf{x}-\hat{\mathbf{x}})(\mathbf{x}-\hat{\mathbf{x}})^{H}\right] \\
& =(\text { DHWGT }-\mathbf{I})(\text { DHWGT }-\mathbf{I})^{H}+\mathbf{D C}_{n} \mathbf{D}^{H}
\end{aligned}
$$

Here we assumed that $E\left[\mathbf{x x}^{H}\right]=\mathbf{I}$, means the transmitted data are independent and identically distributed (i.i.d.) and has the unit power. $\mathbf{C}_{n}$ is the equivalent noise covariance matrix given by

$$
\begin{aligned}
\mathbf{C}_{n}= & e\left[\mathbf{n n}^{H}\right] \\
& =e\left[\left(\mathbf{H W} \mathbf{n}_{r}+\mathbf{n}_{d}\right)\left(\mathbf{H W} \mathbf{n}_{r}+\mathbf{n}_{d}\right)^{H}\right] \\
& =\mathbf{H W W} \mathbf{W}^{H}+\mathbf{I}
\end{aligned}
$$

The weight matrix of the optimal linear receiver which minimizes MSE is essentially the Wiener filter given by

$$
\mathbf{D}=\mathbf{T}^{H} \mathbf{G}^{H} \mathbf{W}^{H} \mathbf{H}^{H}\left(\mathbf{H W G T T}{ }^{H} \mathbf{G}^{H} \mathbf{W}^{H} \mathbf{H}^{H}+\mathbf{C}_{n}\right)^{-1}
$$

where $(\cdot)^{-1}$ denotes matrix inversion. Substituting Equation (5) back into Equation (4) and using matrix inversion lemma, we obtain

$$
\mathbf{E}=\left(\mathbf{T}^{H} \mathbf{G}^{H} \mathbf{W}^{H} \mathbf{H}^{H} \mathbf{C}_{n}^{-1} \mathbf{H W G T}+\mathbf{I}\right)^{-1}
$$

The minimum MSE of the signal estimation at the destination can be expressed as

$$
\begin{aligned}
\text { MMSE } & =\operatorname{tr}(\mathbf{E}) \\
= & \operatorname{tr}\left(\left(\mathbf{T}^{H} \mathbf{G}^{H} \mathbf{W}^{H} \mathbf{H}^{H} \mathbf{C}_{n}^{-1} \mathbf{H W G T}+\mathbf{I}\right)^{-1}\right)
\end{aligned}
$$

Thus the source preceding matrix and the relay amplifying matrix optimization problem can be formulated as

$$
\begin{array}{ll}
\min _{\mathbf{T}, \mathbf{W}} & \operatorname{tr}\left(\left(\mathbf{T}^{H} \mathbf{G}^{H} \mathbf{W}^{H} \mathbf{H}^{H} \mathbf{C}_{n}^{-1} \mathbf{H W G T}+\mathbf{I}\right)^{-1}\right) \\
\text { s.t. } & \operatorname{tr}\left(\mathbf{W}\left(\mathbf{G} \mathbf{T} \mathbf{T}^{H} \mathbf{G}^{H}+\mathbf{I}\right) \mathbf{W}^{H}\right) \leq P_{r} \\
& \operatorname{tr}\left(\mathbf{T T}^{H}\right) \leq P_{s}
\end{array}
$$

where $P_{r}$ and $P_{s}$ are the power constraints at RS and BS.

\section{Optimization Algorithms}

\subsection{Iterative Algorithm}

In [7], authors proposed a unified framework for optimizing linear non-regenerative single user multicarrier MIMO relay systems. We extend this algorithm to solve our single user single carrier optimization.

First we denote the singular value decomposition 
(SVD) of the channel between BS and RS $\mathbf{G}$ and the channel between RS and MS $\mathbf{H}$ as

$$
\begin{aligned}
& \mathbf{G}=\mathbf{U}_{g} \boldsymbol{\Lambda}_{g} \mathbf{V}_{g}^{H} \\
& \mathbf{H}=\mathbf{U}_{h} \boldsymbol{\Lambda}_{h} \mathbf{V}_{h}^{H}
\end{aligned}
$$

where the dimensions of $\mathbf{U}_{g}, \boldsymbol{\Lambda}_{g}, \mathbf{V}_{g}$ are $N_{r} \times N_{r}$, $N_{r} \times N_{s}, N_{s} \times N_{s}$, respectively, and the dimensions of $\mathbf{U}_{h}, \boldsymbol{\Lambda}_{h}, \mathbf{V}_{h}$ are $N_{d} \times N_{d}, N_{d} \times N_{r}, N_{r} \times N_{r}$, respectively. We assume that the main diagonal elements of $\boldsymbol{\Lambda}_{g}$ and $\boldsymbol{\Lambda}_{h}$ are arranged in decreasing order. The closed-form of optimal $\mathbf{T}$ and $\mathbf{W}$ in (8) are given by [7]

$$
\begin{gathered}
\mathbf{T}=\mathbf{V}_{g, 1} \boldsymbol{\Lambda}_{t} \\
\mathbf{W}=\mathbf{V}_{h, 1} \boldsymbol{\Lambda}_{w} \mathbf{U}_{g, 1}^{H}
\end{gathered}
$$

where $\boldsymbol{\Lambda}_{t}$ and $\boldsymbol{\Lambda}_{w}$ are $M \times M$ diagonal matrices, $\mathbf{V}_{g, 1}$, $\mathbf{V}_{h, 1}$ and $\mathbf{U}_{g, 1}$ contain the leftmost M columns from $\mathbf{V}_{g}$, $\mathbf{V}_{h}$ and $\mathbf{U}_{g}$, respectively.

Substitute (9)-(12) into (8), the problem is expressed as

$$
\begin{array}{ll}
\min _{\lambda_{w, i}, \lambda_{i, i}} & \sum_{i=1}^{M}\left(1+\frac{\left(\lambda_{h, i} \lambda_{w, i} \lambda_{g, i} \lambda_{t, i}\right)^{2}}{\left(\lambda_{h, i} \lambda_{w, i}\right)^{2}+1}\right)^{-1} \\
\text { s.t. } & \sum_{i=1}^{M} \lambda_{w, i}^{2}\left(\left(\lambda_{g, i} \lambda_{t, i}\right)^{2}+1\right) \leq P_{r} \\
& \sum_{i=1}^{M} \lambda_{t, i}^{2} \leq P_{s}
\end{array}
$$

where $\lambda_{g, i}, \lambda_{h, i}, \quad i=1, \ldots, M$ denotes the $i$-th diagonal element of $\boldsymbol{\Lambda}_{g 1}$ and $\boldsymbol{\Lambda}_{h 1}$, which contain the largest $M$ elements in $\boldsymbol{\Lambda}_{g}$ and $\boldsymbol{\Lambda}_{h}, \lambda_{w, i}, \lambda_{t, i}$ are the main diagonal elements of $\boldsymbol{\Lambda}_{w}$ and $\boldsymbol{\Lambda}_{t}$, respectively. For the above results, it follows that the non-regenerative MIMO relay single user system becomes equivalent to a set of parallel single-input single-out (SISO) channels [8].

Since the problem (13) is non-convex, the global optimal solution is hard to obtain. In [9], a grid searchbased algorithm is designed to find the global optimal solution for a multicarrier SISO relay system with the MMI criterion. However, the computational complexity of the algorithm in [9] is extremely high, since in order to obtain a reasonably good solution, search over a highdense grid must be employed. In the following, we provide a numerical method [7] to obtain a local-optimal and which has a much lower computational complexity than that of [9].

To simplify notations, let us define

$$
\begin{gathered}
a_{i}=\lambda_{g, i}^{2}, \quad b_{i}=\lambda_{h, i}^{2} \\
x_{i}=\lambda_{t, i}^{2}, \quad y_{i}=\lambda_{w, i}^{2}\left[\left(\lambda_{g, i} \lambda_{t, i}\right)^{2}+1\right], \quad i=1, \ldots, M
\end{gathered}
$$

Then the problem (13) can be equivalently rewritten as

$$
\begin{array}{ll}
\min _{x_{i}, y_{i}} & \sum_{i=1}^{M} \frac{1+a_{i} x_{i}+b_{i} y_{i}}{1+a_{i} x_{i}+b_{i} y_{i}+a_{i} b_{i} x_{i} y_{i}} \\
\text { s.t. } & \sum_{i=1}^{M} y_{i} \leq P_{r} \\
& \sum_{i=1}^{M} x_{i} \leq P_{s}
\end{array}
$$

From (15), we see that the problem is symmetric in $x_{i}$ and $y_{i}, i=1, \ldots, M$, and the power constraints are decomposed. Thus, we can efficiently update $x_{i}$ and $y_{i}$ in an alternating way. First with fixed $y_{i}, i=1, \ldots, M$, we can update $x_{i}$ by solving

$$
\begin{array}{ll}
\min _{x_{i}} & \sum_{i=1}^{M} \frac{1+a_{i} x_{i}+b_{i} y_{i}}{1+a_{i} x_{i}+b_{i} y_{i}+a_{i} b_{i} x_{i} y_{i}} \\
\text { s.t. } & \sum_{i=1}^{M} x_{i} \leq P_{s}
\end{array}
$$

This is a convex optimization problem, using KKT conditions we can obtain

$$
x_{i}=\frac{1}{a_{i}}\left(\sqrt{\frac{a_{i} b_{i} y_{i}}{\mu\left(1+b_{i} y_{i}\right)}}-1\right)^{+}
$$

where $(\cdot)^{+}=\max (\cdot, 0)$, and $\mu$ is the solution to the source power constraint.

In a similar way, we can update $y_{i}$ with given $x_{i}$. Moreover, $x_{i}$ and $y_{i}$ are symmetric, so we can easily get

$$
y_{i}=\frac{1}{b_{i}}\left(\sqrt{\frac{a_{i} b_{i} x_{i}}{v\left(1+a_{i} x_{i}\right)}}-1\right)^{+}
$$

where $v$ is the solution to the relay power constraint.

Note that the conditional updates of $x_{i}$ and $y_{i}$ may either decrease or maintain but cannot increase the objective functions in (13). Monotonic convergence of $x_{i}$ and $y_{i}$ follows directly from this observation. After the convergence of the alternating algorithm, $\lambda_{w, i}, \lambda_{t, i}$ can be obtained from (14) as

$$
\lambda_{t, i}=\sqrt{x_{i}}, \quad \lambda_{w, i}=\sqrt{y_{i} /\left(\lambda_{g, i}^{2} x_{i}+1\right)}, \quad i=1, \ldots, M
$$

The procedure of optimizing source preceding matrix $\mathbf{T}$ and relay amplifying matrix $\mathbf{W}$ is described in Table 1.

\subsection{Simplified Algorithm}

The iterative algorithm may still be computationally intensive for practical systems. Note that if the objective function in (15) can be decoupled for $x_{i}$ and $y_{i}$, then the optimization of $x_{i}$ and $y_{i}$ can be independently conducted, since the constraints in (15) are already decoupled for $x_{i}$ and $y_{i}$ [10]. With this inspiration, we make an approximation of the objective function in (15) 
Table 1. Procedure of Iterative Algorithm.

1) Set iterative number $N$, initialize the algorithm with $y_{i}^{(0)}=\mathrm{P}_{r} / M$, set $n=0$;

2) Get the SVD of $\mathbf{G}=\mathbf{U}_{g} \boldsymbol{\Lambda}_{g} \mathbf{V}_{g}^{H}$ and $\mathbf{H}=\mathbf{U}_{h} \boldsymbol{\Lambda}_{h} \mathbf{V}_{h}^{H}$;

3) Using Equation (17) and given $y_{i}^{(n)}$, obtain

$x_{i}^{(n)}=\frac{1}{a_{i}}\left(\sqrt{\frac{a_{i} b_{i} y_{i}^{(n)}}{\mu\left(1+b_{i} y_{i}^{(n)}\right)}}-1\right)^{+} ;$

4) Using Equation (18) and given $x_{i}^{(n)}$, obtain

$y_{i}^{(n+1)}=\frac{1}{b_{i}}\left(\sqrt{\frac{a_{i} b_{i} x_{i}^{(n)}}{v\left(1+a_{i} x_{i}^{(n)}\right)}}-1\right)^{+} ;$

5) if $n \geq N$, then go to step 6 ;

Otherwise, $n=n+1$ and go to step 3 ;

6) Using Equation (19), (11), (12), we finally get the optimal

source precoding matrix $\mathbf{T}$ and relay amplifying matrix $\mathbf{W}$.

$$
\begin{aligned}
& \sum_{i=1}^{M} \frac{1+a_{i} x_{i}+b_{i} y_{i}}{1+a_{i} x_{i}+b_{i} y_{i}+a_{i} b_{i} x_{i} y_{i}} \\
& \approx \sum_{i=1}^{M} \frac{2+a_{i} x_{i}+b_{i} y_{i}}{1+a_{i} x_{i}+b_{i} y_{i}+a_{i} b_{i} x_{i} y_{i}} \\
& =\sum_{i=1}^{M} \frac{1}{1+a_{i} x_{i}}+\frac{1}{1+b_{i} y_{i}}
\end{aligned}
$$

Then, the problem (15) can be decomposed as two problems as follows

$$
\begin{array}{ll}
\min _{x_{i}} & \sum_{i=1}^{M} \frac{1}{1+a_{i} x_{i}} \\
\text { s.t. } & \sum_{i=1}^{M} x_{i} \leq P_{s}
\end{array}
$$

and

$$
\begin{array}{ll}
\min _{y_{i}} & \sum_{i=1}^{M} \frac{1}{1+b_{i} y_{i}} \\
\text { s.t. } & \sum_{i=1}^{M} y_{i} \leq P_{r}
\end{array}
$$

Both problems (21) and (22) are convex optimization problem, using KKT conditions, we can easily get

$$
x_{i}=\frac{1}{a_{i}}\left(\sqrt{\frac{a_{i}}{\mu}}-1\right)^{+}, \quad y_{i}=\frac{1}{b_{i}}\left(\sqrt{\frac{b_{i}}{v}}-1\right)^{+}, \quad i=1, \ldots, M
$$

where $\mu$ and $v$ are the solution to the constraints in (21) and (22).

The procedure of this optimization is listed in Table 2.

Compare with Table 1, this is a non-iterative algorithm and suboptimal because of the approximation introduced in (20). Therefore, the proposed algorithm has a substantially reduced computational complexity. Note that the approximation (20) is tight when the transmis- sion power $P_{s}$ and $P_{r}$ are sufficiently high. Meanwhile in Section IV we will find that this simplify algorithm yields only a slight MSE and BER increment compared with the iterative algorithm Therefore, the proposed simplified algorithm is very useful for practical relay communication systems.

Table 2. Procedure of simplified algorithm.

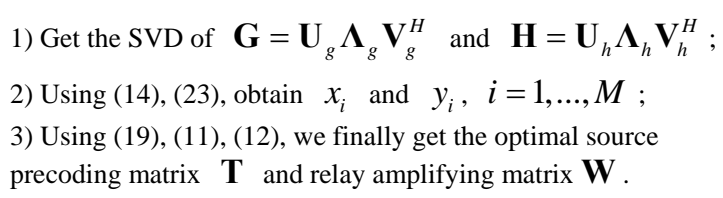

\section{Numerical Results}

In this section, we study the performance of the two-hop non-regenerative MIMO relay systems without direct link. For all examples, we assume the elements of all the channels are i.i.d complex Gaussian with zero mean and unit variance. We also assume that the power constraints at source and relay are the same, i.e. $P_{s}=P_{r}$.

We compare the iterative optimal algorithm and the simplified optimal algorithm in Section 3 (denoted as Simplify I), another simplified optimal algorithm proposed in [11] (denoted as Simplify II), and the naive AF optimal algorithm (NAF). For the NAF relaying, we set the source preceding matrix with the average power allocation to be $\mathbf{T}=\sqrt{P_{s} / M} \mathbf{I}$ and the relay amplifying matrix to be $\mathbf{W}=\sqrt{P_{r} / \operatorname{tr}\left(\mathbf{G T T} \mathbf{T}^{H} \mathbf{G}^{H}+\mathbf{I}\right)} \mathbf{I}$.

We choose $N_{s}=N_{r}=N_{d}=M=2$ in example 1, and $N_{s}=M=4, N_{r}=N_{d}=6$ in example 2. Figure 2 and Figure 3 display the two examples of the MSE of different algorithms versus transmit power from $0 \mathrm{~dB}$ to $40 \mathrm{~dB}$. It can be seen that the iterative algorithm consistently yields the lowest MSE over the whole power range. The performance of the two simplified algorithms is very close to the iterative algorithm. Since for practical communication systems the BER is an important criterion, the performance of all algorithms of example 2 in terms of BER versus transmit power is shown in Figure 4. The QPSK constellations are used. In the simulation, after the estimated vector is obtained by the linear MMSE receiver, a symbol-by-symbol demodulation is used to retrieve the source bits. Note the two simplified algorithms have slightly higher MSE and BER than the iterative algorithm at low SNR. But at high SNR, the three algorithms have almost the same performance. It is reasonable because in the simplified algorithm, the approximate objections are near the original one at high SNR. Considering the computation complexity, the simplified algorithm is very useful for practical systems. 


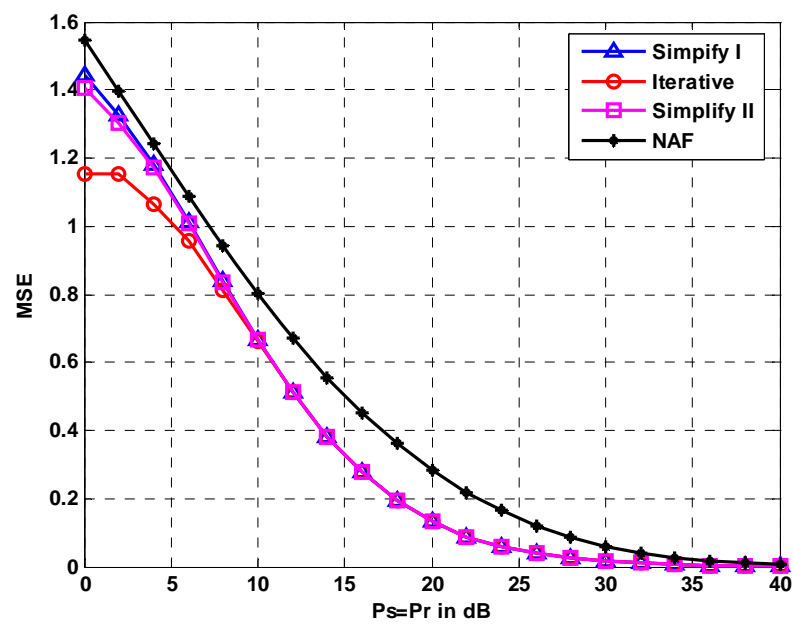

Figure 2. $\mathrm{MSE}$ versus Power, $\mathrm{Ns}=\mathrm{Nr}=\mathrm{Nd}=\mathrm{M}=2$.

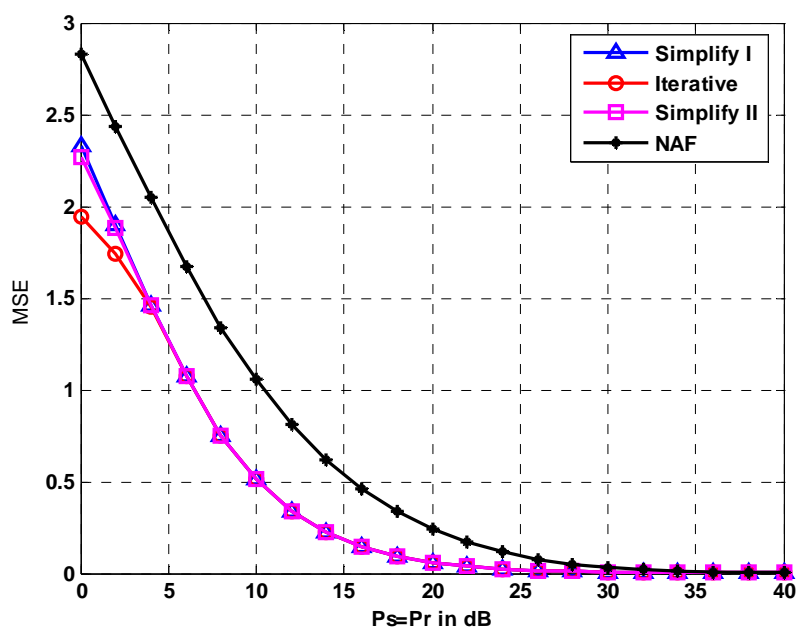

Figure 3. MSE versus Power, $\mathrm{Ns}=4, \mathrm{Nr}=6, \mathrm{Nd}=6, \mathrm{M}=4$.

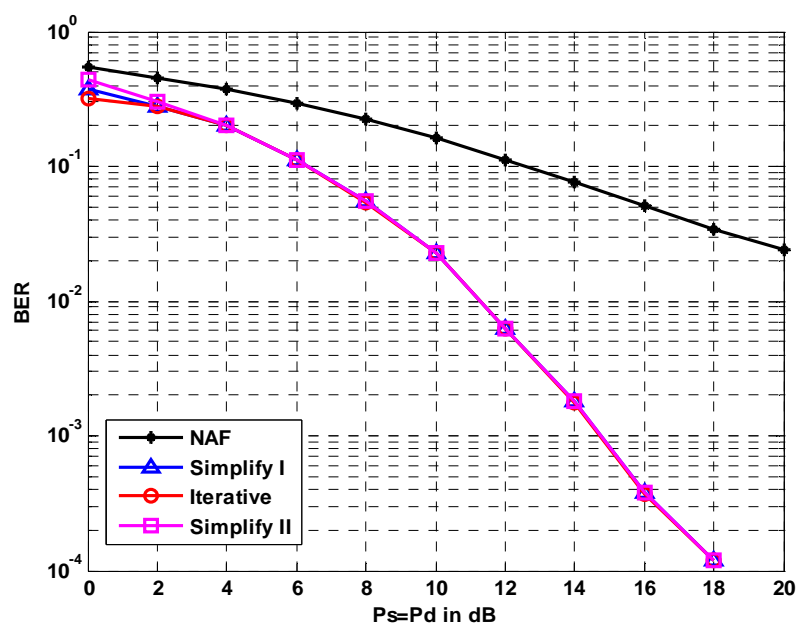

Figure 4. BER versus Power, $\mathrm{Ns}=4, \mathrm{Nr}=6, \mathrm{Nd}=6, \mathrm{M}=4$.

\section{Conclusions}

This paper discussed the optimal design of single user
MIMO relay systems. A number of key architectures has been reviewed and investigated under MMSE criterion. We proposed two different algorithms to find the optimal processing matrix for system. Simulation results showed that the simplified optimal algorithm has slightly higher MSE and BER than the iterative algorithm at low SNR. But at high SNR, both of them have almost the same performance.

\section{REFERENCES}

[1] Y. Hua, "An Overview of Beamforming and Power Allocation for MIMO Relays,” Proceedings of the 2010 Military Communications Conference, Oct. 31-Nov. 3, 2010, pp. 99-104.

[2] B. Wang, J. Zhang and A. Host-Madsen, "On the Capacity of MIMO Channels,” IEEE Transactions. Information Theory, Vol. 51, No. 1, 2005, pp. 29-43. doi:10.1109/TIT.2004.839487

[3] O. Oyman and A. J. Paulraj, "Design and Dnalysis of Linear Distributed MIMO Relaying Algorithms,” IEE Proceedings Communications, Vol. 153, No. 4, 2006, pp. 565-572.doi:10.1049/ip-com:20050406

[4] O. Munoz-Medina, J. Vidal and A. Agustin, "Linear Transceiver Design in Nonregenerative Relays with Channel State Information,” IEEE Tranactions. Signal Processing, Vol. 55, No. 6, 2007, pp.2593-2604. doi:10.1109/TSP.2006.890913

[5] X. J. Tang and Y. B. Hua, "Optimal Design of Non-regenerative MIMO Wireless Relays,” IEEE Transactions Wireless Communications, Vol. 6, No. 4, 2007, pp. 13981407. doi:10.1109/TWC.2007.348336

[6] W. Guan and H. Luo, "Joint MMSE Transceiver Design in Nonregenerative MIMO Relay Systems,” IEEE Communications Letters, Vol. 12, No. 7, 2008, pp.517-519. doi:10.1109/LCOMM.2008.080339

[7] Y. Rong, X. Tang and Y. Hua, “A Unified Framework for Optimizing Linear Non-regenerative Multicarrier MIMO Relay Communication Systems," IEEE Transactions Signal Processing, Vol. 57, No. 12, 2009, pp. 4837-4851. doi:10.1109/TSP.2009.2027779

[8] L. Sanguinetti, A. A. D’Amico and Y. Rong, “A Tutorial on Transceiver Design for Amplify-and-forward MIMO Relay Systems,” IEEE J. Sel. Areas Commun., Vol. 30, 2012.

[9] W. Zhang, U. Mitra and M. Chiang, "Optimization of Amplify-and-forward Multicarrier Two-hop Transmission,” IEEE Transactions Communications, Vol. 59, No. 5, 2011, pp. 1434-1445. doi:10.1109/TCOMM.2011.022811.100017

[10] Y. Rong, "Linear Non-regenerative Multicarrier MIMO Relay Communications Based on MMSE Criterion," IEEE Transactions Communications, Vol. 58, No. 7, 2010, pp. 1918-1923.

[11] Y. Rong, "Simplified Algorithms for Optimizing Multi User Multi-hop MIMO Relay Systems,” IEEE Transactions Communications, Vol. 59, No.10, 2011, pp. 28962904. doi:10.1109/TCOMM.2011.081111.100756 